\title{
Dynamic instability analysis of single walled Carbone NanoTubes conveying fluid under generalized boundary conditions
}

\author{
A. Azrar ${ }^{1}$, L. Azrar ${ }^{1,2}$, and A. A. Aljinaidi ${ }^{2}$ \\ ${ }^{1}$ Department of Mathematics Faculty of Sciences and Techniques of Tangier, Abdelmalek Essaâdi University; Tangier; \\ Morocco \\ ${ }^{2}$ Department of Mechanical Engineering, Faculty of Engineering, King Abdulaziz University, Jeddah, Saudi Arabia
}

\begin{abstract}
The dynamic instabilities of Carbon NanoTubes (CNTs) conveying fluid are modeled and numerically simulated based on the nonlocal elasticity theory. The small scale parameter and the fluid-tube interaction effects on the dynamic behaviors of the CNT-fluid system as well as the instabilities induced by the fluid-velocity are investigated. The critical fluid-velocity and frequency-amplitude relationships as well as the flutter and divergence instability types and the associated time responses can be obtained based on the presented methodological approach.
\end{abstract}

\section{Introduction}

Carbon nanotubes (CNTs) have become one of the most promising material and appear to possess extraordinary physical properties. Many applications of CNTs have been reported, such as in atomic force microscopes (AFMs), sensors, actuators, resonators, nano oscillators and field emission devices. To realize the potential benefits of CNTs a fundamental understanding of nano-structured material is required in order to develop reliable constitutive models for various design purposes. The theory of nonlocal elasticity, Eringen, [1], allows accounting for the small scale effect that is very significant when dealing with micro and nanostructures. Lee and Chang [2], studied the vibration analysis of a viscousfluid-conveying single-walled carbon

nanotube embedded in an elastic medium. Chang and Lee [3] discussed the analysis of the vibration characteristic of fluid-conveying double-walled carbon nanotubes. Khosravian and Rafii-Tabar [4] proposed computational modeling of a non-viscous fluid flow in a multi-walled carbon nanotube using classical boundary conditions. Azrar et al $[5,6]$ developed higher order free vibration analyses of single walled carbon nanotubes with various boundary condition types.

In this paper, the small scale parameter and the internal fluid interaction effects on the dynamic behaviors of the single walled CNT-fluid system as well as the instabilities induced by the fluid-velocity are investigated. Generalized boundary conditions described by translational and rotational springs at both ends are used. These conditions allow describing the more realistic boundary conditions and covering the classical boundary conditions by simply specifying the spring constants. A numerical procedure is elaborated for solutions of the resulting hardl nonlinear equations. The critical fluid-velocity and frequencyamplitude relationships are given the corresponding instability types and time responses are obtained.

\section{Mathematical formulation}

Let us consider a slender single walled carbon nanotube of length $\mathrm{L}$, diameter $\mathrm{d}$ and thickness $\mathrm{h}$ under arbitrary boundary conditions. The nonlocal elasticity theory combined with the Timoshenko beam model is adopted. The dynamic governing equations are formulated and the frequency domain procedure is elaborated.

\subsection{Governing dynamic equations}

Based on the nonlocal elasticity theory, developed by Eringen [1], the constitutive equation for a linear homogenous nonlocal elastic body is given by the following integral equation:

$$
\begin{aligned}
& \sigma_{i j}(x)=\int_{V} \alpha\left(\left|x-x^{\prime}\right|, \tau\right) C_{i j k l} \varepsilon_{k l}\left(x^{\prime}\right) d V\left(x^{\prime}\right), \\
& \varepsilon_{k l}=\left(u_{k, l}+u_{l, k}\right) / 2
\end{aligned}
$$

in which $\alpha\left(\left|x-x^{\prime}\right|, \tau\right)$ is the nonlocal kernel function, which incorporates the nonlocal effect at the reference point $\mathrm{x}$ produced by local strain point at the source $\mathrm{x}$ ' into 
the constitutive equations; $\tau=e_{0} a / L \quad$ where a and $e_{0}$ are a constant appropriate to each material, an internal characteristic length and $\mathrm{L}$ is the length. $C_{i j k l}$ is the elastic modulus tensor, $\sigma_{i j}$ and $\varepsilon_{i j}$ are the stress and the strain tensors. The nonlocal kernel function $\alpha$ depends on the internal and external characteristics lengths. Various approximate models of nonlocal elasticity can be obtained by specifying the kernel function $\alpha$. Based on the Timoshenko beam model the axial strains are:

$\varepsilon_{x x}=\varepsilon_{x x}^{0}+z \frac{\partial \psi}{\partial x}, \varepsilon_{x x}^{0}=\frac{\partial u}{\partial x}, \quad \gamma_{x z}=\psi-\frac{\partial w}{\partial x}$,

where $\psi$ denotes the rotation of the cross section, $u$ and $w$ are the middle plane $(\mathrm{z}=0)$ axial and transverse displacements and $t$ is time. Using these equations, the nonlocal resultant axial force $N$, moment $M$ and shear force $Q$ are given by the following differential equations [5].

$$
\begin{aligned}
& N-\left(e_{0} a\right)^{2} \frac{\partial^{2} N}{\partial x^{2}}=E A \varepsilon_{x x}^{0}, \\
& M=\left(e_{0} a\right)^{2} \frac{\partial^{2} M}{\partial x^{2}}+E I \frac{\partial \psi}{\partial x}, \\
& Q=\left(e_{0} a\right)^{2} \frac{\partial^{2} Q}{\partial x^{2}}+k s A G\left(\psi-\frac{\partial w}{\partial x}\right),
\end{aligned}
$$

in which

$$
N=\int_{A} \sigma_{x x} d A, \quad M=\int_{A} z \sigma_{x x} d A, Q=k s \int_{A} \sigma_{x z} d A
$$

where $k s$ is the factor of shear depending on the shape of the cross section $A$ and its value is $k s=2(1+v) /(4+3 v)$.

I, $E$ and $G$ are the second moment of inertia of the nanotube, Young and shear modulus respectively. The equations of motion for the transversely vibrations of CNT are given by:

$$
\begin{aligned}
& \frac{\partial Q(x, t)}{\partial x}+q(x, t)=\left[\left(m_{c}+m_{f}\right) \frac{\partial^{2} w(x, t)}{\partial t^{2}}\right. \\
& \left.+m_{f} v^{2} \frac{\partial^{2} w(x, t)}{\partial x^{2}}+2 m_{f} v \frac{\partial^{2} w(x, t)}{\partial x \partial t}\right], \\
& \frac{\partial M(x, t)}{\partial x}-Q(x, t)=\left(J_{c}+J_{f}\right) \frac{\partial^{2} \psi(x, t)}{\partial t^{2}},
\end{aligned}
$$

where $q(x, t)$ is the transverse excitation force per unit length and $\mathrm{v}$ is the uniform mean flow velocity of conveying fluid. For a constant cross section, the mass inertia $m_{c}$ and $m_{f}$ are the mass per unit length for CNT and fluid respectively, $J_{c}$ and $J_{f}$ are the mass moment and inertia for CNT and fluid respectively. Substituting Eqs. (4-7) into (8) and (9) one obtains the partial differential system governing the dynamic behavior of undamped CNTs conveying fluid.

$$
\left\{\begin{array}{l}
E I\left(\partial^{2} \psi / \partial x^{2}\right)-k s A G(\psi-\partial w / \partial x) \\
=\partial^{2} / \partial t^{2}\left[\left(J_{c}+J_{f}\right) \psi-J_{c}\left(e_{0} a\right)^{2}\left(\partial^{2} \psi / \partial x^{2}\right)\right] \\
k s A G(\partial / \partial x)(\psi-(\partial w / \partial x))+q-\left(e_{0} a\right)^{2}\left(\partial^{2} q / \partial x^{2}\right) \\
+\partial^{2} / \partial t^{2}\left[\left(m_{0}+m_{f}\right) w-m_{0}\left(e_{0} a\right)^{2}\left(\partial^{2} w / \partial x^{2}\right)\right] \\
+m_{f} v^{2} \partial^{2} w / \partial x^{2}+2 m_{f} v \partial^{2} w / \partial x \partial t=0
\end{array}\right.
$$

\subsection{Frequency domain solution}

For free vibrations, harmonic motion is assumed $w(x, t)=W(x) e^{i \omega t}, \psi(x, t)=\Psi(x) e^{i \omega t}$ and $q(x, t)=0$

where $\omega$ is the natural vibration frequency parameter. For dimensionless equations, the following variables are used.

$y=x / L ; W=w / L ; \tau=L / d ; n_{u}=e_{0} a / L ;$

$$
m=\frac{m_{f}}{\left(m_{c}+m_{f}\right)} ; V=\sqrt{\frac{m_{f} v L}{E I}} ; \beta=\omega \sqrt{\frac{\left(m_{c}+m_{f}\right) L^{4}}{E I}}
$$

The resulting coupled differential equations are then obtained.

$$
\left\{\begin{array}{l}
\frac{d}{d y}\left(\Psi-\frac{d W}{d y}\right)+\left(V^{2} \chi+(1-m) \chi \beta^{2} n_{u}^{2}\right) \frac{d^{2} W}{d y^{2}} \\
+2 i \chi \beta V \sqrt{m} \frac{d W}{d y}-\chi \beta^{2} W=0 \\
(\chi-\zeta) \frac{d^{2} \Psi}{d y^{2}}=\left(\Psi-\frac{d W}{d y}\right)-\delta \chi \beta^{2} \Psi
\end{array}\right.
$$

where $\chi=\frac{4+3 v}{8 \tau^{2}}, \zeta=\frac{(1-\mathrm{m}) \beta^{2} \mathrm{n}_{\mathrm{u}}^{2}}{4 \tau^{2}}$

and $\delta=1 / 4 \tau^{2}(1-\mathrm{m} / 2)$

Eliminating $W$ or $\Psi$ from eqs. (14) and (15) yields to the following uncoupled differential equations:

$$
\begin{aligned}
& (1-\zeta)\left[\left(1-\chi V^{2}-4 \chi \tau^{2} \zeta\right) \frac{d^{4} W}{d y^{4}}-2 i \chi \beta V \sqrt{m} \frac{d^{3} W}{d y^{3}}\right] \\
& +\left[\beta^{2}(\chi(1-\zeta)+\delta)+\left(V^{2}+4 \tau^{2} \zeta\right)\left(1-\delta \chi \beta^{2}\right)\right] \frac{d^{2} W}{d y^{2}} \\
& -2 i \chi \beta V \sqrt{m}\left(\delta \chi \beta^{2}-1\right) \frac{d W}{d y}+\beta^{2}\left(\delta \chi \beta^{2}-1\right) W=0 \\
& (1-\zeta)\left[\left(1-\chi V^{2}-4 \chi \tau^{2} \zeta\right) \frac{d^{4} \Psi}{d y^{4}}-2 i \chi \beta V \sqrt{m} \frac{d^{3} \Psi}{d y^{3}}\right] \\
& +\left[\beta^{2}(\chi(1-\zeta)+\delta)+\left(V^{2}+4 \tau^{2} \zeta\right)\left(1-\delta \chi \beta^{2}\right)\right] \frac{d^{2} \Psi}{d y^{2}} \\
& -2 i \chi \beta V \sqrt{m}\left(\delta \chi \beta^{2}-1\right) \frac{d W}{d y}+\beta^{2}\left(\delta \chi \beta^{2}-1\right) \Psi=0
\end{aligned}
$$

The characteristic equation associated to (16) is given by: 


$$
\begin{aligned}
& (1-\zeta)\left[\left(1-\chi V^{2}-4 \chi \tau^{2} \zeta\right) \lambda^{4}+2 \chi \beta V \sqrt{m} \lambda^{3}\right] \\
& -\left[\beta^{2}(\chi(1-\zeta)+\delta)+\left(V^{2}+4 \tau^{2} \zeta\right)\left(1-\delta \chi \beta^{2}\right)\right] \lambda^{2} \\
& +2 \chi \beta V \sqrt{m}\left(\delta \chi \beta^{2}-1\right) \lambda+\beta^{2}\left(\delta \chi \beta^{2}-1\right)=0
\end{aligned}
$$

The transverse displacement and rotation solutions of (14, 15) are given by:

$$
W(y)=\sum_{n=1}^{4} A_{n} e^{i \lambda_{n} y} ; \Psi(x)=\sum_{n=1}^{4} D_{n} e^{i \lambda_{n} y} ;
$$

where the complex roots $\lambda_{n}(\mathrm{n}=1,2,3,4)$ are determined by solving the nonlinear algebraic equation (18) with respect to $\lambda$ and $A_{n}, D_{n}$ are constants to be determined using the considered boundary conditions. Substituting equation (19) into (15), a relationship between $A_{n}$ and $D_{n}$ is obtained. The associated moment and shear force are then given by:

$$
\begin{aligned}
& M(y)=-\frac{E I}{L}\left[\lambda_{n} \xi_{n}\left(1-n_{u}{ }^{2} \frac{1-m}{4 \tau^{2}} \beta^{2}\right)+n_{u}{ }^{2}(1-m) \beta^{2}\right] W(y) \\
& Q(y)=\frac{E I}{L^{2}} i\left[\left(\xi_{n}+\lambda_{n}\right) / \chi-n_{u}{ }^{2} \lambda_{n}(1-m) \beta^{2}\right] W(y)
\end{aligned}
$$

where the unknown frequency parameter $\beta$ and the arbitrary constants $A_{n}$ will be numerically determined for the considered boundary conditions.

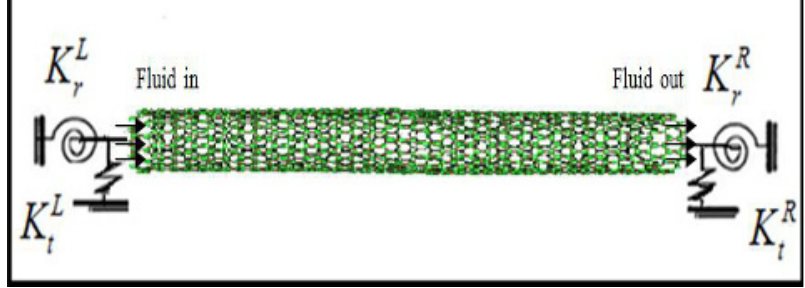

Fig. 1. Elastically restrained CNT at both ends.

The following generalized boundary conditions are considered:

$$
\begin{aligned}
& M^{T}(0)-K_{r}^{L} \frac{d W(0)}{d y}=0 \\
& M^{T}(1)+K_{r}^{R} \frac{d w(1)}{d y}=0 \\
& V^{T}(0)+K_{t}^{L} W(0)=0 \\
& V^{T}(1)-K_{t}^{R} w(1)=0
\end{aligned}
$$

where $K_{r}^{L}, K_{t}^{L}$ and $K_{r}^{R}, K_{t}^{R}$ are the translational and the rotational spring constants at the left and right ends of the CNT at $\mathrm{y}=0$ and $\mathrm{y}=1$, see "figure 1". The classical boundary conditions can be simply obtained as special cases when the stiffness's of the springs take some extreme values such as zero and infinity. For example, the clamped-clamped boundary condition can be easily obtained by assuming that at each end the translational and rotational spring constants are extremely large.

Based on the previous mathematical formulations and on some mathematical developments, the following nonlinear algebraic transcendental equation is obtained: $\left(\alpha_{3} \vartheta_{4} \gamma_{1} \kappa_{2}-\alpha_{3} \vartheta_{4} \gamma_{2} \kappa_{1}-\alpha_{4} \vartheta_{3} \gamma_{1} \kappa_{2}+\alpha_{4} \vartheta_{3} \gamma_{2} \kappa_{1}\right) \mathrm{e}^{\left(\mathrm{i} \lambda_{1}\right)} \mathrm{e}^{\left(\mathrm{i} \lambda_{2}\right)}$

$+\left(-\alpha_{2} \vartheta_{4} \gamma_{1} \kappa_{3}+\alpha_{2} \vartheta_{4} \gamma_{3} \kappa_{1}+\alpha_{4} \vartheta_{2} \gamma_{1} \kappa_{3}-\alpha_{4} \vartheta_{2} \gamma_{3} \kappa_{1}\right) \mathrm{e}^{\left(\mathrm{i} \lambda_{1}\right)} \mathrm{e}^{\left(\mathrm{i} \lambda_{3}\right)}$

$+\left(\alpha_{1} \vartheta_{4} \gamma_{2} \kappa_{3}-\alpha_{1} \vartheta_{4} \gamma_{3} \kappa_{2}-\alpha_{4} \vartheta_{1} \gamma_{2} \kappa_{3}+\alpha_{4} \vartheta_{1} \gamma_{3} \kappa_{2}\right) \mathrm{e}^{\left(\mathrm{i} \lambda_{2}\right)} \mathrm{e}^{\left(\mathrm{i} \lambda_{3}\right)}$

$+\left(\alpha_{2} \vartheta_{3} \gamma_{1} \kappa_{4}-\alpha_{2} \vartheta_{3} \gamma_{4} \kappa_{1}-\alpha_{3} \vartheta_{2} \gamma_{1} \kappa_{4}+\alpha_{3} \vartheta_{2} \gamma_{4} \kappa_{1}\right) \mathrm{e}^{\left(\mathrm{i} \lambda_{1}\right)} \mathrm{e}^{\left(\mathrm{i} \lambda_{4}\right)}$

$+\left(-\alpha_{1} \vartheta_{3} \gamma_{2} \kappa_{4}+\alpha_{1} \vartheta_{3} \gamma_{4} \kappa_{2}+\alpha_{3} \vartheta_{1} \gamma_{2} \kappa_{4}-\alpha_{3} \vartheta_{1} \gamma_{4} \kappa_{2}\right) \mathrm{e}^{\left(\mathrm{i} \lambda_{2}\right)} \mathrm{e}^{\left(\mathrm{i} \lambda_{4}\right)}$

$+\left(\alpha_{1} \vartheta_{2} \gamma_{3} \kappa_{4}-\alpha_{1} \vartheta_{2} \gamma_{4} \kappa_{3}-\alpha_{2} \vartheta_{1} \gamma_{3} \kappa_{4}+\alpha_{2} \vartheta_{1} \gamma_{4} \kappa_{3}\right) \mathrm{e}^{\left(\mathrm{i} \lambda_{3}\right)} \mathrm{e}^{\left(\mathrm{i} \lambda_{4}\right)}=0$

where $\alpha_{j}, \vartheta_{j}, \gamma_{j}, \kappa_{j}$ are given by:

$\alpha_{j}=U_{1 j}+K_{t}^{L} ; \vartheta_{\mathrm{j}}=U_{2 j}-i K_{r}^{L} \lambda_{j} ; \gamma_{\mathrm{j}}=U_{1 j}-K_{t}^{R} ;$

$\kappa_{\mathrm{j}}=U_{2 j}+i K_{r}^{R} \lambda_{j}, j=1,2,3,4$.

in which $\mathrm{U}_{1 \mathrm{j}}, \mathrm{U}_{2 \mathrm{j}}$ are given by:

$U_{1 j}=\frac{E I}{L}\left[-\lambda_{j} \xi_{j}\left(1-n_{u}{ }^{2} \frac{1-m}{4 \tau^{2}} \beta^{2}\right)+n_{u}{ }^{2}(1-m) \beta^{2}\right] ;$

and $U_{2 j}=\frac{E I}{L^{2}}\left[i \chi\left(\xi_{j}+\lambda_{j}\right)+i n_{u}^{2}(1-m) \beta^{2} \lambda_{j}\right]$;

Note that these coefficients depend on the fluid-velocity

$\mathrm{V}$, the small scale parameters $\mathrm{n}_{\mathrm{u}}$ as well as on other physical and material properties of the considered CNT.

The numerical solution of the latter nonlinear equation allows getting the natural frequencies with respect to the fluid-velocity and CNT physical and material parameters. The resulting mode deflection and rotation shapes are given by:

$W(\mathrm{y})=A_{1} \mathrm{e}^{i \lambda_{1} y}+A_{2} \mathrm{e}^{i \lambda_{2} \mathrm{y}}+A_{3} \mathrm{e}^{i \lambda_{3} \mathrm{y}}+\mathrm{e}^{i \lambda_{4} \mathrm{y}} ;$
$\Psi(\mathrm{y})=i \xi_{n}\left(A_{1} \mathrm{e}^{i \lambda_{1} y}+A_{2} \mathrm{e}^{i \lambda_{2} \mathrm{y}}+A_{3} \mathrm{e}^{i \lambda_{3} \mathrm{y}}+\mathrm{e}^{i \lambda_{4} \mathrm{y}}\right) ;$

where

$$
\begin{aligned}
& A_{1}=-\left[\left(\alpha_{3} \vartheta_{4} \gamma_{2}-\alpha_{4} \vartheta_{3} \gamma_{2}\right) \mathrm{e}^{\left(\mathrm{i} \lambda_{2}\right)}-\left(\alpha_{2} \vartheta_{4} \gamma_{3}-\alpha_{4} \vartheta_{2} \gamma_{3}\right) \mathrm{e}^{\left(\mathrm{i} \lambda_{3}\right)}\right. \\
&\left.+\left(\alpha_{2} \vartheta_{3} \gamma_{4}-\alpha_{3} \vartheta_{2} \gamma_{4}\right) \mathrm{e}^{\left(\mathrm{i} \lambda_{4}\right)}\right] /\left[\left(\alpha_{2} \vartheta_{3} \gamma_{1}-\alpha_{3} \vartheta_{2} \gamma_{1}\right) \mathrm{e}^{\left(\mathrm{i} \lambda_{1}\right)}\right.\left.-\left(\alpha_{1} \vartheta_{3} \gamma_{2}-\alpha_{3} \vartheta_{1} \gamma_{2}\right) \mathrm{e}^{\left(\mathrm{i} \lambda_{2}\right)}+\left(\alpha_{1} \vartheta_{2} \gamma_{3}-\alpha_{2} \vartheta_{1} \gamma_{3}\right) \mathrm{e}^{\left(\mathrm{i} \lambda_{3}\right)}\right] \\
& A_{2}= {\left[\left(\alpha_{3} \vartheta_{4} \gamma_{1}-\alpha_{4} \vartheta_{3} \gamma_{1}\right) \mathrm{e}^{\left(\mathrm{i} \lambda_{1}\right)}-\left(\alpha_{1} \vartheta_{4} \gamma_{3}-\alpha_{4} \vartheta_{1} \gamma_{3}\right) \mathrm{e}^{\left(\mathrm{i} \lambda_{3}\right)}\right.} \\
&+\left.\left(\alpha_{1} \vartheta_{3} \gamma_{4}-\alpha_{3} \vartheta_{1} \gamma_{4}\right) \mathrm{e}^{\left(\mathrm{i} \lambda_{4}\right)}\right] /\left[\left(\alpha_{2} \vartheta_{3} \gamma_{1}-\alpha_{3} \vartheta_{2} \gamma_{1}\right) \mathrm{e}^{\left(\mathrm{i} \lambda_{1}\right)}\right. \\
&\left.-\left(\alpha_{1} \vartheta_{3} \gamma_{2}-\alpha_{3} \vartheta_{1} \gamma_{2}\right) \mathrm{e}^{\left(\mathrm{i} \lambda_{2}\right)}+\left(\alpha_{1} \vartheta_{2} \gamma_{3}-\alpha_{2} \vartheta_{1} \gamma_{3}\right) \mathrm{e}^{\left(\mathrm{i} \lambda_{3}\right)}\right] \\
& \text { and } A_{3}=-\left[\gamma_{4} \mathrm{e}^{\left(\mathrm{i} \lambda_{4}\right)}+\frac{\gamma_{1} \mathrm{e}^{\left(\mathrm{i} \lambda_{1}\right)}\left(\alpha_{2} \vartheta_{4}-\alpha_{4} \vartheta_{2}\right)}{\left(\vartheta_{2}-\alpha_{2} \vartheta_{1}\right)}\right. \\
&\left.-\frac{\gamma_{2} \mathrm{e}^{\left(\mathrm{i} * \lambda_{2}\right)}\left(\alpha_{1} \beta_{4}-\alpha_{4} \vartheta_{1}\right)}{\left(\alpha_{1} \vartheta_{2}-\alpha_{2} \vartheta_{1}\right)}\right] /\left[\gamma_{3} \mathrm{e}^{\left(\mathrm{i} \lambda_{3}\right)}\right] \\
&\left.+\frac{\gamma_{1} \mathrm{e}^{\left(\mathrm{i} \lambda_{1}\right)}\left(\alpha_{2} \vartheta_{3}-\alpha_{3} \vartheta_{2}\right)}{\left(\alpha_{1} \vartheta_{2}-\alpha_{2} \vartheta_{1}\right)}-\frac{\gamma_{2} \mathrm{e}^{\left(\mathrm{i} \lambda_{2}\right)}\left(\alpha_{1} \vartheta_{3}-\alpha_{3} \vartheta_{1}\right)}{\left(\alpha_{1} \vartheta_{2}-\alpha_{2} \vartheta_{1}\right)}\right]
\end{aligned}
$$

Equations (26-28) give the mathematical modeling of the free vibration of CNT conveying fluid under generalized boundary conditions. The fluid-velocity as well as the small length scale and generalized boundary conditions effects on the eigenfrequencies and eigenmodes can be analyzed. The divergence and flutter instabilities and the corresponding responses with respect to the fluid-velocity and generalized boundary conditions are numerically investigated. 


\section{Numerical results and discussions}

Numerical results are presented using the following geometrical and material properties.

$a=1.42 A^{\circ}, h=0.066 \mathrm{~nm}, E=5.5 T P a, v=0.19$,

$d=0.678 \mathrm{~nm}, \rho_{f}=1 \mathrm{~g} / \mathrm{cm}^{3}, m_{f}=2.94 \times 10^{-16} \mathrm{~kg} / \mathrm{m}$,

$\rho_{s}=2.3 \mathrm{~g} / \mathrm{cm}^{3}, m_{c}=3.23 \times 10^{-16} \mathrm{~kg} / \mathrm{m}, J_{f}=1.69 \times 10^{-35} \mathrm{kgm}$,

$J_{c}=3.72 \times 10^{-35} \mathrm{kgm}, V_{n}=V_{0} \sqrt{\rho_{s} / E}$,

In this paper, a numerical procedure based on the NewtonRaphson iterative algorithm has been elaborated for numerical solutions. Various numerical results such as frequency-fluid-velocity curves and the corresponding deflection responses are obtained. The critical velocities corresponding to the divergence and flutter instabilities are obtained for various types of boundary conditions.

The simply supported (S-S) case is first considered. It has to be noted the classical sine modes, usually considered, lead to erroneous results in this case. A well adapted approach, as presented here, is needed for accurate results. The evolutions of the real and imaginary components of dimensionless frequency parameter with the dimensionless flow velocity are presented in "figure 2" for the first three modes of an S-S CNT. The S-S condition is obtained by simply $\operatorname{choosing}\left(K_{t}^{R}=K_{t}^{L}=0, K_{r}^{L}=K_{r}^{R}=10^{9}\right)$. The effect of these stiffnesses as well as of the aspect ratio L/d and small scale length ' $\mathrm{e}_{0} \mathrm{a}$ ' on the critical velocities can be investigated.

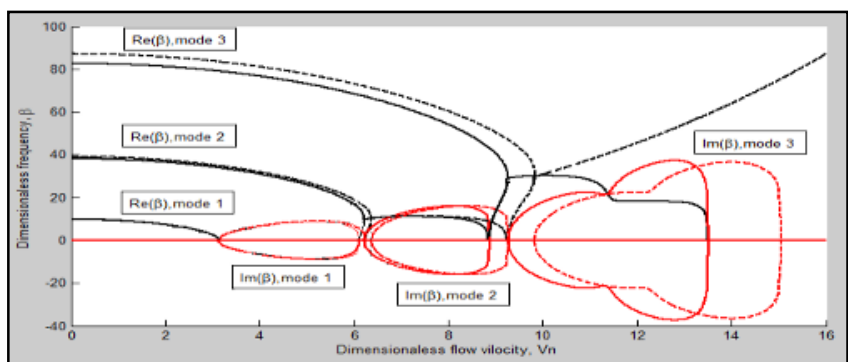

Fig. 2 Real and imaginary components of dimensionless frequency $\beta$ as a function of flow velocity $\mathrm{V}$ for the first three modes of a simply supported CNT with different aspect ratios $\mathrm{L} / \mathrm{d}$ and $\mathrm{e}_{0} \mathrm{a} / \mathrm{L}=0.05$. Solid line $\mathrm{L} / \mathrm{d}=20$, dashed line $\mathrm{L} / \mathrm{d}=40$.

"Figure 2" shows the flutter and divergence velocities for the considered S-S CNT. The real frequency parts increases with increasing of the aspect ratio $\mathrm{L} / \mathrm{d}$. In addition, as the aspect ratio increases, the flow velocity of vibration induced flutter instability of CNT increases. The divergence in the first mode occurs at critical velocity $\mathrm{V}_{\mathrm{c}} \mathrm{d}^{\mathrm{d}}=3.3$ and in the second mode at $\mathrm{V}_{\mathrm{c}}{ }^{\mathrm{d}}=6.3$. The flutter instabilities occurs at $\mathrm{V}_{\mathrm{c}}{ }^{\mathrm{F}}=6.4$ and $\mathrm{V}_{\mathrm{c}}{ }^{\mathrm{F}}=9.2$ and the coalescence between the first-second and the second-third modes are respectively obtained. It is observed that only the real part of $\beta_{1}$ (first frequency parameter) vanishes completely as the flow velocity reaches $\mathrm{V}_{\mathrm{c}}^{\mathrm{d}}$, while the imaginary part becomes nonzero. Physically, the first mode becomes unstable by flutter instability when the flow velocity is higher than $\mathrm{V}_{\mathrm{c}} \mathrm{F}^{\mathrm{F}}=6.3$.

The other boundary conditions can be easily recovered by simply choosing the used spring stiffness's. "Figure 3", shows the variations of the real and imaginary components of $\beta_{1}$ associated to the first mode shape for a CNT with the flow velocity for various rotational springs $\left(K_{r}^{L}=K_{r}^{R}=0,10,50,100,10^{9}\right)$. Note that by this way the edges change from S-S to clamped (C-C). It can be seen that the boundary conditions have a strong effect on the critical velocities and then on the instability types. Some other numerical results are obtained for time responses at different velocity levels and spring stiffness's values.

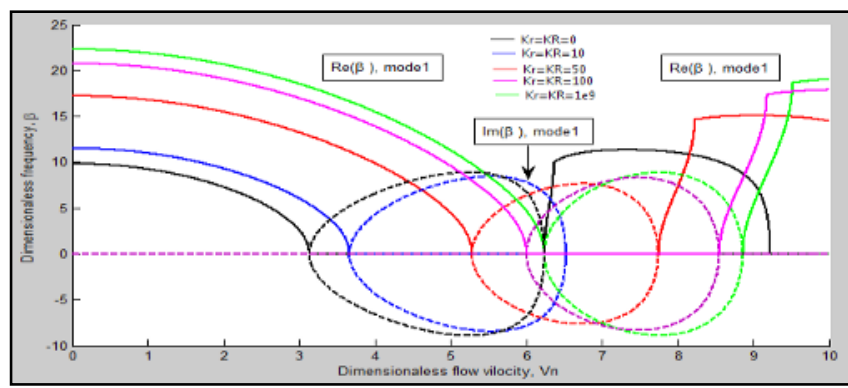

Fig.3 Real and imaginary components of the frequency parameter $\beta$ as a function of flow velocity $\mathrm{V}$ for the first mode of a CNT under various boundary conditions, $\left(K_{t}^{L}=K_{t}^{R}=10^{9}\right)$ with $\left(K_{r}^{L}=K_{r}^{R}=0,10,50,100,10^{9}\right)$

\section{4- Conclusion}

In this paper a mathematical modeling and a methodological approach are developed for the dynamic instability analysis of CNT conveying fluid. The small scale parameter and the generalized boundary conditions accounting for a more realistic and large wide of boundary conditions are considered. Based on the analysis, it was observed that the divergence instability occurs first and then the flutter one at more large fluid-velocity. The aspect ratio $\mathrm{L} / \mathrm{d}$ effect becomes significant at large flow velocity and the boundary conditions have strong effects on the critical velocities and instability types.

\section{Acknowledgements}

The authors would like to acknowledge the financial support provided by the convention CNRST-CNRS (Morocco -French), SPM 11/11 and from the DSR research project at King Abdulaziz University in Jeddah, SA.

\section{References}

[1] A.C. Eringen, J. Appl. Phys. 54, 4703, (1983).

[2] H. L. Lee and W. J. Chang, J. Physica E 41, 529, (2009).

[3] T. Natsuki, Q. Q. Ni and M. Endo, J. Applied Physics 105, 094328, (2009).

[4] A. Azrar, L. Azrar and A. A. Aljinaidi, J. Physica E. (to be published)

[5] A. Azrar, L. Azrar And A. A. Aljinaidi, Revue de Mécanique Théorique et Appliquée (to be published)

[6] N Khosravian and H Rafii-Tabar, J. of Nanotechnology 19, 9, (2008). 